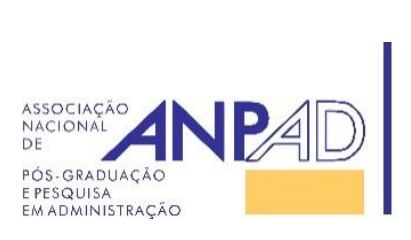

Disponível em

http://www.anpad.org.br/rac

RAC, Rio de Janeiro, v. 20, n. 2, art. 3, pp. 175-196, Mar./Abr. 2016

http://dx.doi.org/10.1590/1982-7849rac2016140057

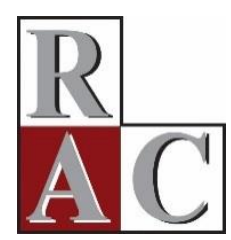

(c) EY $^{\circ}$

\title{
Atitudes e Habilidades Sociais para Trabalho em Equipe: Desenvolvimento de uma Escala
}

\author{
Social Skills and Attitudes for Teamwork: Scale Development
}

Catarina Cecília Odelius ${ }^{1}$ Rafael Nishino Ono ${ }^{1}$

Gardênia da Silva Abbad ${ }^{1}$ Pedro Henrique Melo Albuquerque ${ }^{1}$

Universidade de Brasília ${ }^{1}$ 


\title{
Resumo
}

Este estudo objetivou desenvolver uma escala de medida com foco em componentes afetivos de competências (atitudes e habilidades sociais) para Trabalho em Equipe (AHTE), em grupos de pesquisa. A escala foi desenvolvida com base em pesquisas anteriores e nos estudos de Odelius e Sena (2009) e Odelius et al. (2011). Os dados foram coletados por meio de entrevistas, que submetidas à análise de conteúdo, subsidiaram a elaboração de questionário. Este, após ser submetido à análise semântica e teórica, foi testado e depois respondido por 396 participantes de grupos de pesquisa. A análise fatorial policórica revelou o agrupamento das competências em quatro fatores (abertura à diversidade humana, habilidades sociais, cooperação, responsabilidade), responsáveis por $55 \%$ da variância total explicada, com índice de confiabilidade $\alpha=0,96$. Os resultados são parcialmente semelhantes aos realizados em outros contextos. Ao final, são apontadas direções para realização de novas pesquisas.

Palavras-chave: abertura a diversidade humana; habilidades sociais; grupos de pesquisa; análise fatorial policórica.

\begin{abstract}
A scale for measurement of Teamwork Attitudes and Skills (TAS) of research groups (components of competencies) is developed in this study, based on previous research and studies by Odelius and Sena (2009) and Odelius et al. (2011). Data were collected by means of interviews and questionnaires. Interview data were submitted to content analysis, and then used to develop a questionnaire. This questionnaire was submitted to judges for theoretical and semantic validation and was pre-tested. It was then answered by 396 participants of research groups. Factor analysis of ordinal variables revealed four competency factors (openness to human diversity; social skills, cooperation, and responsibility) that represent 55\% of total variance explained, with reliability $\alpha=0.96$. The results are partially similar to those carried out in other contexts. Directions for further research are indicated at the end of the paper.
\end{abstract}

Key words: openness to human diversity; social skills; research groups; polychoric factor analysis. 


\section{Introdução}

A literatura reconhece um aumento do interesse a respeito dos temas grupos de pesquisa (Erdmann \& Lanzoni, 2008; Hamui Sutton, 2010a) e competências (Brandão, 2007, 2008; Godoy, Antonello, Bido, \& Silva, 2009), indicando diferentes focos das pesquisas relativas a essas temáticas.

Grupos de pesquisa são descritos como produtores de conhecimento (Gaviria Velásquez, Mejía Correa, \& Henao Henao, 2007; Odelius et al., 2011) e como um local que promove o desenvolvimento dos participantes dos grupos (Hamui Sutton, 2010a, 2010b). Diferentes autores indicam que os grupos proporcionam e incentivam a realização de novos trabalhos (Moura, Leader, Pelletier, \& Abrams, 2008), potencializam as relações entre fatores contextuais e a aprendizagem do indivíduo por meio do trabalho em equipe (Williams, Scandura, \& Gavin, 2009), além de permitir aplicações práticas dos resultados das pesquisas (Riquelme \& Langer, 2010). Outros estudos abordam o modo de organização dos grupos e suas características (Du, 2009; Mauthner \& Doucet, 2008; Millward, Banks, \& Riga, 2010); fatores que afetam os grupos de pesquisa em relação a sua dinâmica de funcionamento e a sua produtividade (Barbosa, Sasso, \& Berns, 2009; Erdmann \& Lanzoni, 2008; Pepe et al., 2010); benefícios gerados pela participação (Abma, Nierse, \& Widdershoven, 2009; Nierse, Schipper, Zadelhoff, Griendt, \& Abma, 2011) e modelos de análise de grupos com a intenção de analisar desempenho (González-Alcaide, Aleixandre-Benavent, \& Granda-Orive, 2010; Restrepo \& Villegas, 2007; Rizo \& Eduardo, 2010; Weijden, Gilder, Groenewegen, \& Klasen, 2008).

Já os estudos relativos a competências têm abordado, entre outros, as correntes teóricas adotadas por diferentes autores (Brandão, 2007; Dutra, 2004; Jones \& Moore, 2008; Leitão, 2010), níveis de objeto de estudo (Brandão \& Guimarães, 2001; King, Fowler, \& Zeithaml, 2002), desenvolvimento de competências (Antonello, 2006; Bahry \& Tolfo, 2007; Godoy et al., 2009; Zarifian, 2001), relação entre competências e aprendizagem (Garavan \& McGuire, 2001), competência no trabalho (McMullan et al., 2003), variáveis associadas a competências (Bowditch \& Buono, 1992; Le Boterf, 2003; Martin-Baró, 1983; Skinner, 1987; Todorov, 1989; Zarifian, 2001) e características e problemas identificados nesse campo de estudo (Brandão \& Borges-Andrade, 2007; Garavan \& McGuire, 2001; Godoy \& Antonello, 2009; Jubb \& Robotham, 1997).

Estudos que tratam conjuntamente os temas grupos de pesquisa e competências são relativamente poucos e abordam a competência como: fator motivador para os participantes de grupos de pesquisa (Alon, 2010); gerador de inovação e melhoria no processo de pesquisa (Higuita-López, Molano-Velandia, \& Rodríguez-Merchán, 2011); espaço de aprendizagem colaborativa, (Barrachina, Sanz-Torrent, \& Serrat-Sellabona, 2009) e desenvolvimento de competências individuais necessárias para a atuação em grupos de pesquisa (Haythornthwaite, 2006; Odelius, Abbad, Resende, Sena, \& Ono, 2010; Odelius et al., 2011; Odelius \& Sena, 2009).

Entre os estudos que focam o desenvolvimento de competências, o de Odelius et al. (2011) ressalta a importância da realização de novos estudos, com a elaboração e validação de questionário com base nas categorias de conteúdo identificadas e que abrangem: (a) competências técnicas relacionadas à análise e revisão de literatura, leitura e interpretação de textos, realização de pesquisa e processamento de dados (trabalhar com banco de dados, utilizar software estatístico); (b) competências de comunicação, como capacidade de ouvir o outro, expor ideias em público, redigir textos científicos; (c) comportamentos e atitudes, como disciplina, ética, motivação, pró-atividade, versatilidade, raciocínio lógico, interação social (abertura à ideia do outro, capacidade de trabalhar em equipes, relacionamento interpessoal); e, de modo mais acentuado para os líderes, (d) aspectos relacionados à gestão (visão estratégica, visão sistêmica, gestão de pessoas e de recursos).

Considerando essa recomendação e que uma escala de medida de competências técnicas foi desenvolvida por Fernandez e Odelius (2013), o presente estudo objetivou elaborar e identificar evidências de validade de uma escala de medida com foco em componentes afetivos de competências (atitudes e habilidades sociais) para trabalho em equipe em grupos de pesquisa, dando continuidade a estudos anteriores. 
O desenvolvimento dessa escala poderá viabilizar a identificação do domínio de componentes afetivos de competências em grupos de pesquisa e possibilitar o seu aprimoramento, conforme sugerido por Higuita-López, Molano-Velandia e Rodríguez-Merchán (2011), assim como o estudo da relação dessa variável com outras, tais como características do grupo, desempenho e uso de estratégias de aprendizagem.

O trabalho está estruturado em cinco tópicos, além desta introdução. O referencial teórico, inicialmente, caracteriza grupos de pesquisa e, na sequência, aborda o tema competências, apresentando uma breve contextualização do tema, aspectos que o constituem com foco especial em conteúdos relativos a habilidades, comportamentos, atitudes, valores, interação, comunicação e competências específicas relativas a grupos de pesquisa. Os procedimentos metodológicos estão abordados em tópicos específicos, seguidos dos resultados, discussão e conclusão.

\section{Grupos de Pesquisa}

Os grupos de pesquisa são formados por professores com diferentes cargos no plano de carreira e por pesquisadores de diferentes níveis de formação (estudantes de iniciação científica, graduandos em conclusão do curso, mestrandos, doutorandos e pós-doutorandos) sob a tutela de um orientador que lhes atribui tarefas e acompanha seus trabalhos (Alvarez \& Vidal, 2001). Esses autores defendem que os grupos de pesquisa são os elementos que permitem conectar um sistema pesquisa-universidade, com o intuito de atender às demandas que são apresentadas pelas agências de fomento.

Os grupos de pesquisa também são descritos como produtores de conhecimento e considerados como um ambiente que proporciona o entendimento da forma pela qual a pesquisa deve ser realizada (Gaviria Velásquez et al., 2007). Além disso, eles são notados como um local que promove o desenvolvimento de seus participantes quanto a hábitos, formas de agir e de realizar pesquisas que norteiam as expectativas e o tipo de construção de conhecimento cultivado (Hamui Sutton, 2010a, 2010b).

Em relação à criação dos grupos de pesquisa, alguns são projetados para atender ao compromisso social de formar indivíduos. Já outros são incentivados pelas necessidades de recursos e/ou pela busca de cargos e empregos para seus integrantes (Riquelme \& Langer, 2010).

Grupos de pesquisa são identificados como ambientes propícios ao desenvolvimento de competências coletivas (habilidades interpessoais necessárias ao trabalho em equipe, atitudes favoráveis à solução colaborativa de problemas, respeito à diversidade humana), geralmente pouco estimuladas em situações formais e tradicionais de ensino-aprendizagem (Odelius et al., 2011).

\section{Competências}

Garavan e McGuire (2001) destacam que estudos a respeito de competências apresentam uma multiplicidade de abordagens, o que origina inúmeros desafios e limitações. Os autores abordam problemas filosóficos e epistemológicos de estudo do tema, em especial, a falta de consenso em relação a definições, a dificuldade de avaliar e mensurar a existência de competências e a de efetuar uma classificação das mesmas. O tema é estudado sob várias perspectivas (comportamentalista, cognitivista e construtivista), abordando diferentes características (individuais, organizacionais e como ferramenta para estruturar e facilitar a comunicação entre educação e mercado de trabalho), com diferentes focos (demandas para o indivíduo, demandas do trabalho, ou ambos) e abordando diferentes conteúdos.

A competência, vista como característica subjacente ao indivíduo, é usualmente relacionada ao o desempenho bem-sucedido na realização de uma tarefa ou em determinada situação (McClelland, 1973; 
McMullan et al., 2003), podendo ainda fazer referência ao padrão de desempenho atingido (o que as pessoas fizeram) ou ao comportamento pelo qual o desempenho é alcançado (como as pessoas fazem) (Rowe, 1995).

Bergenhenegouwen, Horn e Mooijman (1997) e McMullan et al. (2003) ressaltam que, quando as competências estão relacionadas ao trabalho, correspondem a descrições de ações, ou comportamentos ou resultados que uma pessoa deve demonstrar com o seu desempenho.

Em relação aos aspectos que constituem as competências, não há consenso na literatura. Para Parry (1996), competência refere-se a um conjunto de conhecimentos, habilidades e atitudes que se relacionam e afetam a maior parte de um trabalho, um papel ou responsabilidade e que permite o desempenho de tarefas com certos níveis de proficiência.

Já para Bergenhenegouwen et al. (1997), a competência humana se estrutura em quatro níveis, que apresentam diferentes patamares de complexidade: (a) conhecimentos e habilidades, (b) habilidades intermediárias, (c) valores e padrões éticos e morais e (d) auto-imagem, motivos, esforços, entusiamo e persuasão. Por sua vez, Cheetham e Chivers (1996, 1998, 2005), ao definirem um modelo de desenvolvimento de competências profissionais, estabelecem quatro grupos de competências essenciais (cognitivas, funcionais, pessoais/comportamentais, éticas/valores) e meta-competências, que se subdividem em outros aspectos constituintes. As respectivas definições destas competências e seus conteúdos estão apresentados na Tabela 1.

Tabela 1

Definições de Competências e Respectivos Aspectos Abordados, de acordo com Modelo de Cheetham e Chivers (1996, 1998, 2005)

\begin{tabular}{|c|c|c|}
\hline Competências & Definição & Aspectos constituintes \\
\hline $\begin{array}{l}\text { Cognitivas/ } \\
\text { Conhecimento }\end{array}$ & $\begin{array}{l}\text { Possessão de conhecimentos apropriados } \\
\text { relativos ao trabalho e habilidade em } \\
\text { colocá-los em uso efetivo }\end{array}$ & $\begin{array}{l}\text { Conhecimentos: } \\
\text { Tácito e prático } \\
\text { Técnico e teórico } \\
\text { Procedimental } \\
\text { Contextual }\end{array}$ \\
\hline Funcionais & $\begin{array}{l}\text { Habilidade de desempenhar efetivamente as } \\
\text { tarefas do trabalho e produzir resultados } \\
\text { específicos. }\end{array}$ & $\begin{array}{l}\text { Específicos da ocupação } \\
\text { Organizacional e processos } \\
\text { Mental } \\
\text { Psicomotor }\end{array}$ \\
\hline $\begin{array}{l}\text { Pessoais/ } \\
\text { comportamentais }\end{array}$ & $\begin{array}{l}\text { Habilidade para adotar comportamentos } \\
\text { apropriados, observáveis em situações } \\
\text { relativas ao trabalho. }\end{array}$ & $\begin{array}{l}\text { Social/ vocacional: comportamentos } \\
\text { relacionados ao desempenho das tarefas } \\
\text { profissionais: auto-confiança, centralidade } \\
\text { na tarefa; resistência, etc. }\end{array}$ \\
\hline Éticas/valores & $\begin{array}{l}\text { Possessão de valores pessoais e } \\
\text { profissionais apropriados e habilidade em } \\
\text { utilizá-los para realizar julgamentos a } \\
\text { respeito de situações relativas ao trabalho. }\end{array}$ & $\begin{array}{l}\text { Pessoal (ex.: aderência a códigos morais e } \\
\text { religiosos; aderência à legislação) } \\
\text { Profissional (ex: aderência a códigos } \\
\text { profissionais; centralidade no cliente; } \\
\text { sensitividade ambiental; adoção de atitudes } \\
\text { profissionais apropriadas; dever de se } \\
\text { manter atualizado) }\end{array}$ \\
\hline
\end{tabular}




\section{Tabela 1 (continuação)}

\begin{tabular}{lll}
\hline Competências & Definição & Aspectos constituintes \\
\hline Meta-competências & São competências que estão além das & Comunicação \\
& demais competências, podendo auxiliar na & Auto-desenvolvimento \\
& aquisição ou análise das outras & Criatividade \\
& competências & Análise \\
& & Solução de problemas \\
& & Reflexão (1998) \\
& Agilidade Mental (1998) \\
\hline
\end{tabular}

Nota. Fonte: elaborada pelos autores com base nos dados de Cheetham, G., \& Chivers, G. (1996). Towards a holistic model of professional competence. Journal of European Industrial Training, 20(5), 20-30. doi: 10.1108/03090599610119692; Cheetham, G., \& Chivers, G. (1998). The reflective (and competente) practitioner. A model of professional competence with seeks to harmonise the reflective practitioner and competence based approaches. Journal of European Industrial Training, 22(7), 267-276; Cheetham, G., \& Chivers, G. (2005). Professions, competence and informal learning. Cheltenham, UK: Edward Elgar.

Para Bergenhenegouwen et al. (1997), os conhecimentos e habilidades estão associadas a ações mais instrumentais relacionadas à realização adequada de uma tarefa, sendo passíveis de aprendizado por meio de treinamento, enquanto que as habilidades intermediárias são aplicáveis a várias situações e são a base para o desenvolvimento de outras qualificações, sendo mais difíceis de serem aprendidas, demandando supervisão e feedback. As habilidades intermediárias incluem, entre outras: habilidades comunicativas e sociais; abertura a novas ideias, que consiste em aproveitar as ideias de outros para incrementar o próprio desempenho; o trabalhar em equipe, que diz respeito à contribuição para o desempenho da equipe e à tomada de decisão, procurando compartilhar experiência com outros integrantes; a aceitação de responsabilidade, desempenhando atividades sem a necessidade de supervisão, buscando prevenir problemas. Já os valores e padrões éticos e morais demandam a internalização por meio de experiências e processos de socialização, abrangendo visão de mundo e/ou das outras pessoas, opiniões específicas a respeito de culturas, valores e tradições. As competências relacionadas à auto-imagem, motivos, esforços, entusiamo e persuasão, apesar de serem mais difíceis de serem observadas, são a base de todas as demais.

A aprendizagem de fatos e comportamentos, a aprendizagem social, a verbal e a conceitual e de procedimentos estão entre os conteúdos associados ao desenvolvimento de competências humanas (Pozo, 2002).

Para Le Boterf (2003), as habilidades são criadas e/ou desenvolvidas a partir da utilização dos conhecimentos em situações de trabalho, de aprendizagem ou de ensino e podem ser: formais, como condutas, métodos ou instrumentos em que o indivíduo possui domínio; empíricas, resultantes das lições adquiridas de experiências na prática; relacionais (social); e cognitivas. Durand (1997), por sua vez, define habilidade como a capacidade de agir de uma maneira concreta, seguindo procedimentos ou objetivos predefinidos. Já as atitudes são internalizadas e se revelam pela manifestação de habilidades sociais.

As atitudes, na definição de Gagné e colaboradores são

estados complexos do ser humano que afetam o comportamento em relação a pessoas, coisas e eventos, determinando a escolha de um curso de ação pessoal. O efeito da atitude é justamente ampliar a reação positiva ou negativa de uma pessoa, ou seja, sua predisposição, em relação a algo (Gagné e colaboradores como citado em Freitas \& Brandão, 2006, p. 100).

As atitudes são, portanto, estados mentais adquiridos que influenciam o indivíduo a escolher a ação na qual se engajará (Gagné \& Medsker, 1996). Envolvem crenças e valores pessoais, embora estes sejam mais gerais, enquanto atitudes são mais específicas e orientadas para determinadas finalidades e objetos (Abbad \& Borges-Andrade, 2014). 
Apesar de todas as competências humanas serem desenvolvidas em contextos sociais, as habilidades sociais são as que possuem conteúdos específicos associados à boa comunicação, a comportamentos de interação com pessoas e objetos, a atitudes que possibilitam ao indivíduo avaliar objetos, pessoas, fatos, situações e escolher o modo de agir, bem como a representações sociais culturalmente compartilhadas (Pozo, 2002).

As habilidades sociais, de acordo com definição de Del Prette e Del Prette (2006, p. 1), são "diferentes classes de comportamentos sociais no repertório do indivíduo, para lidar de maneira adequada com as demandas das situações interpessoais" e incluem assertividade, autoestima, comunicação, autoexposição e autocontrole da agressividade.

Godoy, Antonello, Bido e Silva (2009), tomando como referência o modelo de Cheetham e Chivers e a realização de análise fatorial, identificaram que as competências adquiridas durante o curso de graduação em administração se organizam em quatro construtos: competência social, competência para solução de problemas, competência técnico-profissional e competências básicas, sendo que estas podem ser consideradas como o fator gerador das demais. Nos resultados obtidos, os itens relativos à competência de valores e ética foram eliminados e, das competências básicas (que equivalem às metacompetências), somente emergiram comunicação, análise, raciocínio lógico e reflexão.

Entre os estudos que identificaram competências necessárias e/ou desenvolvidas com a atualização em grupos de pesquisa estão os de Haythornthwaite (2006), Odelius e Sena (2009), HiguitaLópez et al. (2011) e Odelius et al. (2011).

Higuita-López et al. (2011) classificaram as competências requeridas aos grupos de pesquisa em três grandes grupos: competências gerais (que são necessárias a qualquer pessoa, mas relacionadas à atuação em grupo de pesquisa), competências associadas ao processo investigativo e competências relativas à inovação, ou seja, à geração ou melhoria de novos produtos e materiais. Entre as competências emergentes no estudo estão os princípios éticos na forma de proceder, o manejo de idiomas e visão internacional, enquanto que as competências identificadas como mais relevantes aos grupos foram:

Competências gerais: de gestão, trabalho em equipe, planejamento de pesquisa, iniciativa para inovação, abertura para a mudança, liderança, associatividade, manejo de conflitos e gestão de tecnologias;

Competências específicas ao processo de pesquisa: definição de problema de pesquisa, questionamento, hipótese de trabalho, preparação de campo, estado da arte, continuidade nos processos de pesquisa, formação em investigação, interação com outros grupos de pesquisa e gestão de recursos.

Haythornthwaite (2006) identificou que grupos de pesquisa eram propícios ao desenvolvimento de competências técnicas, relativas aos campos específicos de conhecimento, métodos de investigação e tecnologias computacionais; competências relativas ao trabalho em equipe e em colaboração; competências administrativas necessárias ao desenvolvimento de projetos de pesquisa e competências de socialização e de formação de redes sociais.

Entre as competências resultantes da participação em grupos de pesquisa, identificadas nos estudos realizados por Odelius e colaboradores (Odelius \& Sena, 2009; Odelius et al., 2011), estão, além de conhecimentos e habilidades técnicas necessárias ao desenvolvimento de pesquisa, as seguintes competências: aspectos relacionados à gestão, como visão estratégica e visão sistêmica; gestão de pessoas, abrangendo a coordenação de atividades, o controle de diferentes ritmos, a valorização e o aproveitamento das contribuições de integrantes menos instruídos; valores e atitudes favoráveis ao trabalho em equipe e o respeito à diversidade humana, às limitações e aos interesses pessoais; habilidades interpessoais (assertividade para trabalhar com pessoas com características diferentes, trabalho em equipe, relacionamento interpessoal, ponderação de aspectos políticos para tomada de decisão, desenvolvimento de autonomia para resolver problemas e comunicação); habilidades sociais necessárias à socialização profissional; e comportamentos e atitudes, como disciplina, ética, motivação, 
pró-atividade, versatilidade, raciocínio lógico, além da postura adequada para a atuação profissional, a geração de ideias, a socialização e a formação de redes sociais. No item comunicação foi destacada a capacidade de ouvir o outro e a exposição de ideias em público e, na interação social, a abertura à ideia do outro.

É possível identificar, portanto, que há uma diversidade de abordagens e de classificação de competências, sendo que, neste estudo, serão enfatizados os componentes afetivos das competências, segundo definições de diversos autores sobre domínio afetivo (Bloom, Krathwohl, \& Masi, 1974), habilidades sociais (Pozo, 1999), atitudes (Gagné \& Medsker, 1996) e valores e ética (Cheetham \& Chivers, 1996, 1998, 2005).

Em suma, a participação de estudantes em grupos de pesquisa favorece o desenvolvimento de competências essenciais à formação de profissionais capazes de atuar e pesquisar, entre as quais estão competências técnicas e componentes afetivos das competências (atitudes, habilidades sociais e valores) relacionados ao trabalho em equipe. $\mathrm{O}$ grupo de pesquisa, como espaço de aprendizagem colaborativa, assume, pelo exposto, um importante papel formativo, complementar ao ensino tradicional oferecido pelas universidades.

\section{Método}

Este estudo, de acordo com o critério de classificação proposto por Creswell (2010), é classificado como misto. Ele é descritivo exploratório - em decorrência dos métodos quantitativos de análises de dados utilizados, uma vez que os parâmetros associados a cargas fatoriais bem como outras medidas foram estimados, caracterizando um processo de análise descritiva exploratória paramétrica - e aplicado e qualitativo por possuir finalidade prática e poder contribuir para a identificação de competências em grupos de pesquisas.

Em relação aos meios de investigação, foi utilizada análise documental - com levantamento de dados junto a fontes secundárias (CAPES e CNPq) para identificar os grupos de pesquisa de programas de pós-graduação - e pesquisa de campo, com a realização de entrevistas e aplicação de questionários junto a participantes de grupos de pesquisa de universidades.

O processo de construção do instrumento de coleta de dados abrangeu 5 etapas: revisão de literatura; entrevistas com pesquisadores; elaboração de questionário; validação semântica e teórica de instrumentos; e, por fim, teste do instrumento junto à população-alvo.

A revisão de literatura identificou poucos estudos que investigassem competências em grupos de pesquisa e que tivessem realizado pesquisa de campo, tendo os resultados obtidos sido analisados com o intuito de identificar competências desenvolvidas em grupos de pesquisa. Como base para a construção do instrumento de coleta de dados, foi utilizada categoria de competências descrita no estudo de Odelius et al. (2011): atitudes, valores e habilidades para o trabalho em equipe que, de acordo com esses autores, abrange competências do domínio afetivo, habilidades de relacionamento interpessoal, valores e atitudes necessárias ao trabalho em equipe.

$\mathrm{Na}$ segunda etapa, foram realizadas 10 entrevistas individuais com líderes de grupos de pesquisa consolidados em suas áreas de conhecimento, visando a explorar novos achados em relação às competências desenvolvidas com a atuação em grupos de pesquisa. O roteiro semiestruturado solicitava aos líderes a indicação de competências necessárias e desenvolvidas através da atuação no grupo e informações a respeito do funcionamento e características do grupo. Após término da coleta, foi realizada análise de conteúdo, que, segundo Rocha e Deusdará (2005), permite captar um saber que está por trás da superfície textual e é uma técnica neutra de verificação de uma determinada realidade. Foram identificados inicialmente 37 itens que diziam respeito a atitudes, valores e habilidades sociais necessárias e/ou desenvolvidas através da atuação nos grupos, além de outros que se referiam a competências técnicas, que não são objeto deste relato e, portanto, não estão detalhadas. A inclusão de 
itens identificados como necessários e como desenvolvidos através da atuação dos grupos é decorrente do fato de que algumas das competências foram identificadas pelos pesquisados, ora como necessárias, ora como desenvolvidas, dependendo do grupo ao qual o respondente pertencia. Além do mais, as competências desenvolvidas na atuação do grupo são decorrentes de necessidades que se colocam no dia a dia.

O desenvolvimento da escala foi realizado observando recomendações da literatura para validação semântica e teórica de instrumentos (Cassep-Borges, Balbinotti, \& Teodoro, 2010; Pasquali, 2010), visando a melhorar a sua aplicabilidade e assegurar a clareza, a representatividade e a relevância dos itens, ou seja, a sua validação de conteúdo. Buscou-se encontrar falhas ou lacunas no conjunto de itens utilizados para mensurar a categoria atitudes, valores e habilidades para o trabalho em equipe em grupos de pesquisa, bem como detectar a presença de itens que mensuravam outros construtos.

Participaram da validação cinco juízes, que são pesquisadores das áreas de psicologia organizacional e do trabalho, de estudos organizacionais e de gestão de pessoas, com experiência em validação de instrumentos. Para esses juízes, foi enviado um formulário com instruções de preenchimento, bem como uma tabela contendo itens para avaliação e observação quanto a critérios de clareza e objetividade e quanto à adequação dos itens de classificação como atitudes, habilidades e valores para trabalho em equipe.

O mecanismo escolhido para identificar itens não adequados aos objetivos do instrumento foi o coeficiente de validade de conteúdo (CVC) (Cassep-Borges et al., 2010), calculado com base na avaliação dos juízes-avaliadores em relação à:

Clareza de linguagem: considera a linguagem utilizada nos itens, tendo em vista as características da população respondente.

Pertinência prática: considera se cada item foi elaborado de forma a avaliar o conceito de interesse em uma determinada população; analisa se de fato cada item possui importância para o instrumento.

Relevância teórica: considera o grau de associação entre o item e a teoria; visa a analisar se o item está relacionado com o construto.

Os critérios foram aplicados para cada item com escala Likert de 5 pontos, que se caracteriza como uma medida qualitativa ordinal (Stevens, 1946), variando de pouquíssima adequação a muitíssima adequação. Além dos itens contidos na versão preliminar, o instrumento de avaliação continha um campo para anotação das observações para que os juízes-avaliadores expressassem opiniões e/ou sugestões para cada item.

A avaliação realizada por juízes permitiu o aprimoramento do instrumento de coleta de dados, resultando na exclusão, inclusão e alteração de redação de itens e na adequação de conteúdo e de pertinência, conforme avaliação do CVC (itens que obtiveram valores inferiores a 0,8 foram excluídos). $\mathrm{O}$ instrumento resultante ficou com 33 itens relativos a atitudes, valores e habilidades para o trabalho em equipe.

As limitações assumidas para o estudo, nesse processo de desenvolvimento do instrumento, são relativas a não terem sido efetuados o cálculo de erro para avaliação de possível viés dos juízesavaliadores e a análise da concordância entre as avaliações dos juízes quanto à pertinência teórica dos itens.

O questionário incluía também uma apresentação com informações relativas à pesquisa, orientações para resposta, um espaço destinado à coleta de dados demográficos /funcionais, (como idade, gênero, tempo de experiência em pesquisa, ano e mês de ingresso no grupo, escolaridade e situação no grupo), o esclarecimento de que os dados seriam tratados de modo a preservar a identidade dos respondentes e, por fim, os agradecimentos. 
Para a validação semântica, o instrumento também foi aplicado a um grupo de 10 indivíduos formado por estudantes, professores e pesquisadores integrantes de 2 grupos pesquisa de uma mesma área de conhecimento. Esta etapa teve como objetivo verificar se os itens eram compreensíveis para membros da população-alvo (Cassep-Borges et al., 2010; Peres-dos-Santos \& Laros, 2007) e procurou identificar eventuais falhas ou incorreções, eliminando ambiguidades - se essas surgissem - com o intuito de garantir a compreensão dos itens e da escala pela população estudada e evitar erros decorrentes de interpretação do formato. Em decorrência da validação semântica, foram realizados apenas alguns ajustes no enunciado dos itens.

O questionário, em sua versão final, foi aplicado em uma amostra de participantes de grupos de pesquisa. Uma consulta à plataforma Lattes e ao diretório de grupos de pesquisa permitiu identificar líderes com bolsa de produtividade 1A, 1C, 1D e 2, tendo sido solicitado a esses pesquisadores, que atuam em diferentes áreas de conhecimento, que enviassem o link de pesquisa aos participantes dos grupos em que atuavam.

A apresentação da pesquisa destacava o foco da coleta de dados em competências desenvolvidas através da atuação no grupo de pesquisa e foi solicitado aos pesquisados que indicassem que competências foram desenvolvidas a partir da atuação no grupo, informando o grau de domínio que possuíam em cada um dos itens relativos à competência, variando entre 0 (nenhum domínio) a 10 (domínio completo).

Em decorrência do uso da escala de 11 pontos (com âncoras de significados opostos em seus extremos - tipo Osgood), a escala construída é caracterizada como sendo qualitativa ordinal, e, portanto, por não possuir métrica clara, deve-se utilizar abordagem própria para a análise, qual seja, Análise Fatorial Ordinal (AFO). Com esse intuito, uma matriz de correlações policóricas foi estimada e utilizada como insumo para a AFO, seguindo a proposta de Rigdon e Ferguson (1991).

\section{Resultados e Discussão}

O instrumento de coleta de dados foi respondido por 396 participantes de grupo de pesquisa, sendo 178 do sexo feminino, 203 do sexo masculino e 15 que não informaram. Foi observado que a menor idade respondida foi de 18 anos e a maior foi de 81 . A idade média de idade dos respondentes foi de 41 anos com desvio padrão de 14,03, e 18 participantes não responderam suas idades. Em relação à representatividade por região, há pesquisadores que participam de organizações que atuam em mais de uma região, e observa-se predominância de respondentes localizados na região sudeste, que representam $50,4 \%$ do total amostrado, o que é de se esperar, uma vez que há maior concentração de pesquisadores e grupos nessa região. Para o tempo de experiência, foi observado que o menos experiente possui 5 meses de pesquisa e o mais experiente possui 50 anos de pesquisa. De todos os respondentes, 30 não responderam esta informação ou preencheram de forma incorreta, invalidando a resposta. A média de experiência em pesquisa foi de 16,5 anos, com um desvio padrão de 13,7. Em relação ao grau de formação, $62 \%$ da amostra possuem doutorado ou pós-doutorado.

O agrupamento das competências em fatores latentes aos dados foi realizado com o método de Análise Fatorial Ordinal por meio de correlação policórica (Rigdon \& Ferguson, 1991), por ser essa abordagem mais adequada quando se considera dados categóricos ordinais, uma vez que os itens, por serem medidos segundo uma estrutura qualitativa ordinal, não permite que se estimem as variâncias e covariâncias necessárias para a estimação das variáveis latentes por meio da abordagem usual de Análise Fatorial exploratória.

Em uma matriz de correlações (ou variância ou covariâncias) policóricas, uma variável latente contínua com escores politômicos é assumida e suas respostas observadas (variáveis ordinais) são consideradas manifestação dos respondentes que excedem um determinado número de limiares (threshold) latentes. Então, com base nessa matriz, o modelo de Análise Fatorial pode ser aplicado para 
se estimar as variáveis contínuas latentes ao processo de resposta dos entrevistados, em outras palavras, a partir dos dados ordinais, a matriz de correlação policórica estima a relação linear de duas variáveis contínuas latentes observando apenas a informação ordinal.

Nesse trabalho, utilizou-se as propostas de Bentler e Yuan (1996, 1998) e Zoski e Jurs (1993, 1996), com rotação oblimin por meio do software $R$ juntamente com as bibliotecas psych, polycor e nFactors, a qual indicou a retenção de 4 fatores para a matriz de correlações policóricas estimada com base nas respostas dos pesquisados segundo estimador em duas etapas de Olsson (1979).

Quanto ao ajustamento do modelo, o KMO (Kaiser-Meyer-Olkin) do modelo com todos os itens foi superior a 0,85 , o que sugere um bom ajuste do método de Análise Fatorial por meio de correlação policórica; ademais, para o teste de Bartlett, obteve-se 8483.596. Os dados estão apresentados na Tabela 2.

Tabela 2

\section{Análise da Medida KMO e do Teste de Bartlett}

\begin{tabular}{lll}
\hline Estatística & & Valor \\
\hline Kaiser-Meyer-Olkin & &, 91 \\
Teste de esfericidade de Bartlett & Qui Quadrado & 8483.596 \\
& Aprox. & \\
& df & 435 \\
& Sig. &, 000 \\
\hline
\end{tabular}

Nota. Fonte: Dados da pesquisa.

Corrobora-se, assim, o bom ajuste do modelo, uma vez que a hipótese alternativa de fatorabilidade da matriz de dados é aceita.

Na Tabela 3, estão apresentadas a estrutura dos fatores e as comunalidades dos itens. Para a análise das comunalidades, deve ser avaliada a existência de valores extremos, 0 e 1, uma vez que esses valores extremos poderiam indicar patologias associadas ao processo de estimação da matriz de variâncias e covariâncias, como o caso de Heywood (Rindskopf, 1984). Não foram observados valores extremos na Tabela 3, porém foram observados valores baixos para a comunalidade em dois itens: estudar em grupo $(0,27)$ e mobilizar rede de relacionamentos para executar atividades de pesquisa $(0,31)$.

Tabela 3

Representação dos Itens em Fatores e Comunalidade

\begin{tabular}{|c|c|c|c|c|c|}
\hline & Fator $1^{\mathrm{a}}$ & Fator $2^{\mathrm{a}}$ & Fator $3^{\mathrm{a}}$ & Fator $4^{\mathrm{a}}$ & Comunalidade \\
\hline Respeitar a diversidade de opiniões. & 0,84 & & & & 0,77 \\
\hline $\begin{array}{l}\text { Estar disposto a trabalhar com pessoas com } \\
\text { características diferentes. }\end{array}$ & 0,79 & & & & 0,67 \\
\hline Valorizar a diversidade humana. & 0,66 & & & & 0,58 \\
\hline Respeitar valores diferentes. & 0,55 & & & & 0,51 \\
\hline Tratar indistintamente bem as pessoas. & 0,54 & & & & 0,60 \\
\hline Reconhecer a importância de cada pessoa. & 0,52 & & & & 0,70 \\
\hline Respeitar o ritmo de trabalho de cada um. & 0,46 & & & & 0,49 \\
\hline $\begin{array}{l}\text { Aproveitar o feedback dos colegas como } \\
\text { oportunidade de crescimento e aprimoramento. }\end{array}$ & 0,39 & & & & 0,60 \\
\hline
\end{tabular}




\section{Tabela 3 (continuação)}

\begin{tabular}{|c|c|c|c|c|c|}
\hline & Fator $1^{\mathrm{a}}$ & 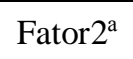 & Fator $^{\mathrm{a}}$ & Fator $4^{\mathrm{a}}$ & Comunalidade \\
\hline $\begin{array}{l}\text { Estar disposto a atuar em equipes } \\
\text { multidisciplinares. }\end{array}$ & 0,38 & & & & 0,44 \\
\hline $\begin{array}{l}\text { Apresentar para o grupo seu ponto de vista de } \\
\text { forma clara e ordenada. }\end{array}$ & & 0,69 & & & 0,55 \\
\hline $\begin{array}{l}\text { Identificar o momento adequado para a } \\
\text { apresentação de novas ideias. }\end{array}$ & & 0,68 & & & 0,50 \\
\hline $\begin{array}{l}\text { Dialogar com pessoas de campos diferentes com a } \\
\text { intenção de identificar outras perspectivas de } \\
\text { solução para determinado problema. }\end{array}$ & & 0,65 & & & 0,46 \\
\hline Expressar ideias sem medo de ser criticado. & & 0,62 & & & 0,48 \\
\hline $\begin{array}{l}\text { Saber me posicionar quando uma situação no } \\
\text { grupo prejudica o desempenho de minhas funções. }\end{array}$ & & 0,60 & & & 0,51 \\
\hline $\begin{array}{l}\text { Expor argumentos de modo convincente, com } \\
\text { desenvoltura. }\end{array}$ & & 0,57 & & & 0,44 \\
\hline $\begin{array}{l}\text { Reelaborar as próprias ideias a partir da apreciação } \\
\text { de propostas apresentadas por outros integrantes } \\
\text { do grupo. }\end{array}$ & & 0,52 & & & 0,47 \\
\hline $\begin{array}{l}\text { Exaurir as possibilidades de encaminhamento de } \\
\text { soluções para problemas que emergem durante as } \\
\text { pesquisas. }\end{array}$ & & 0,51 & & & 0,56 \\
\hline Estudar em grupo. & & 0,47 & & & 0,27 \\
\hline $\begin{array}{l}\text { Resolver conflitos surgidos entre os membros do } \\
\text { grupo de pesquisa. }\end{array}$ & & 0,45 & & & 0,43 \\
\hline $\begin{array}{l}\text { Mobilizar rede de relacionamentos para executar } \\
\text { atividades de pesquisa. }\end{array}$ & & 0,44 & & & 0,31 \\
\hline $\begin{array}{l}\text { Identificar qual papel lhe compete na dinâmica das } \\
\text { relações no grupo (tarefas a realizar e posturas a } \\
\text { assumir). }\end{array}$ & & 0,41 & & & 0,49 \\
\hline Saber a hora de falar e a hora de ouvir o colega. & & 0,30 & & & 0,41 \\
\hline $\begin{array}{l}\text { Saber com quem buscar informações necessárias à } \\
\text { pesquisa. }\end{array}$ & & 0,30 & & & 0,45 \\
\hline $\begin{array}{l}\text { Compartilhar conhecimentos com os demais } \\
\text { integrantes do grupo de pesquisa. }\end{array}$ & & & 0,91 & & 0,81 \\
\hline $\begin{array}{l}\text { Colaborar com outros integrantes na realização de } \\
\text { atividades de pesquisa. }\end{array}$ & & & 0,90 & & 0,84 \\
\hline $\begin{array}{l}\text { Transmitir conhecimentos relevantes, relacionados } \\
\text { a atividades do grupo, aos integrantes do grupo de } \\
\text { pesquisa. }\end{array}$ & & & 0,74 & & 0,73 \\
\hline $\begin{array}{l}\text { Atuar com profissionalismo em todas as situações } \\
\text { relacionadas à pesquisa. }\end{array}$ & & & 0,52 & & 0,53 \\
\hline Buscar colaborativamente a solução de problemas. & & & 0,48 & & 0,58 \\
\hline $\begin{array}{l}\text { Cumprir as obrigações e tarefas sob sua } \\
\text { responsabilidade. }\end{array}$ & & & & 0,80 & 0,76 \\
\hline Ter disciplina para a realização das atividades. & & & & 0,67 & 0,63 \\
\hline
\end{tabular}

Nota. Fonte: Dados da pesquisa.

${ }^{\mathrm{a}}$ Cargas Fatoriais. 
Para a redução do instrumento de coleta de dados, foi feita a avaliação dos itens segundo suas cargas fatoriais, tendo sido excluídos os que apresentaram cargas abaixo de 0,30 e os que apresentaram cargas semelhantes em 2 fatores. Os itens excluídos foram: agir de maneira proativa para resolver problemas, atuar com ética nas atividades de pesquisa e reconhecer as próprias limitações quanto ao domínio do conteúdo (e procurar estudá-lo devidamente quando isso for importante para o grupo). Nos casos em que havia carga fatorial em mais de um fator, com diferenças maiores do que 0,1 , o item foi mantido no fator onde apresentou carga fatorial de maior valor.

É interessante destacar que conteúdo semelhante a atuar com ética nas atividades de pesquisa também foi excluído do estudo de Godoy et al. (2009), apesar da importância reconhecida de que profissionais devem atuar com postura ética e moral de modo a efetuar julgamentos que observem códigos morais e atitudes profissionais apropriadas (Cheetham \& Chivers, 1996, 1998, 2005). O fato de esse item ser sujeito à desestabilidade social, por ser condição necessária à atuação em pesquisa, pode ter contribuído para a exclusão. Para a avaliação de condutas sob o prisma ético, talvez o uso de casos traga dados mais precisos a respeito dos valores éticos dos estudantes.

A exclusão do item relativo à pró-atividade vai em direção contrária a resultados de outras pesquisas (Higuita-López et al., 2011; Odelius \& Sena, 2009) que ressaltam a importância de os profissionais atuarem de modo autônomo e com iniciativa própria visando à solução de problemas e/ou obtenção de resultados e, ainda, o desempenho de atividades sem a necessidade de supervisão, buscando prevenir problemas (Bergenhenegouwen, Horn, \& Mooijman, 1997). Uma possível explicação é a limitação de estudantes serem proativos, uma vez que experimentos ou estudos estão sob a responsabilidade técnica e científica do docente.

O mesmo ocorre com o item reconhecer as próprias limitações quanto ao domínio do conteúdo (e procurar estudá-lo devidamente quando isso for importante para o grupo), que equivale ao item conhecimento e admissão quando não é competente, que integra as metacompetências abordadas por Cheetham e Chivers (1998). O reconhecimento de limitações permite ao indivíduo agir proativamente para desenvolver suas competências.

Pasquali (2008) apresenta uma classificação dos valores das cargas fatoriais para analisar a qualidade dos itens: maior que 0,71 - excelente; entre 0,63 e 0,70 - muito boa; entre 0,55 e 0,62 - boa; entre 0,45 e 0,54 - razoável; abaixo de 0,45 - pobre. Os resultados indicam que $50 \%$ dos itens apresentam carga fatorial entre boas e excelentes. Em função de esta pesquisa ser um estudo exploratório (Richardson, 2010), optou-se pela manutenção dos itens que apresentaram cargas razoáveis e pobres. Recomenda-se cuidado especial em relação a esses itens em estudos posteriores de análise confirmatória.

Os quatro fatores identificados, que correspondem a 55\% da variância total explicada, foram nomeados e definidos de modo a representar o conteúdo dos itens agrupados. Essas informações, juntamente com um resumo de características de cada fator e ordinal coefficient alpha (Zumbo, Gadermann, \& Zeisser, 2007), estão apresentadas na Tabela 4.

Tabela 4

Definições e Características dos Fatores

\begin{tabular}{cllccc}
\hline Fator & Nome & Definição & $\begin{array}{c}\text { Número de } \\
\text { itens }\end{array}$ & $\begin{array}{c}\text { Variação das } \\
\text { cargas fatoriais }\end{array}$ & $\begin{array}{c}\text { Alfa de } \\
\text { Cronbach }\end{array}$ \\
\hline 1 & $\begin{array}{c}\text { Abertura à } \\
\text { diversidade } \\
\text { humana }\end{array}$ & $\begin{array}{l}\text { Refere-se ao modo como as pessoas } \\
\text { interagem com outras pessoas, } \\
\text { respeitando diferenças individuais } \\
\text { de opinião e atuação, identificando e } \\
\text { assimilando diferenças. }\end{array}$ & 9 & 0,38 a 0,84 & 0,96 \\
\hline
\end{tabular}


Tabela 4 (continuação)

\begin{tabular}{|c|c|c|c|c|c|}
\hline Fator & Nome & Definição & $\begin{array}{l}\text { Número de } \\
\text { itens }\end{array}$ & $\begin{array}{l}\text { Variação das } \\
\text { cargas fatoriais }\end{array}$ & $\begin{array}{l}\text { Alfa de } \\
\text { Cronbach }\end{array}$ \\
\hline 2 & $\begin{array}{l}\text { Habilidades } \\
\text { sociais }\end{array}$ & $\begin{array}{l}\text { Refere-se ao grau de assertividade } \\
\text { das pessoas ao se posicionarem na } \\
\text { interação com outras pessoas e nas } \\
\text { situações com as quais se defrontam. }\end{array}$ & 14 & 0,30 a 0,69 & 0,95 \\
\hline 3 & Cooperação & $\begin{array}{l}\text { Refere-se ao grau de atuação } \\
\text { colaborativa, compartilhamento de } \\
\text { conhecimento e atuação conjunta na } \\
\text { realização das atividades de } \\
\text { pesquisa. }\end{array}$ & 5 & 0,48 a 0,91 & 0,92 \\
\hline 4 & Responsabilidade & $\begin{array}{l}\text { Refere-se ao modo como o } \\
\text { indivíduo se posiciona na realização } \\
\text { das atividades, visando ao alcance } \\
\text { de resultados. }\end{array}$ & 2 & 0,67 e 0,80 & 0,88 \\
\hline
\end{tabular}

A correlação entre os fatores está apresentada na Tabela 5.

Tabela 5

\section{Correlação entre os Fatores}

\begin{tabular}{lcccc}
\hline Fator & $\begin{array}{c}\text { Abertura à } \\
\text { diversidade humana }\end{array}$ & Habilidades sociais & Cooperação & Responsabilidade \\
\hline Abertura à diversidade humana & 1 & 1 & & \\
Habilidades sociais & 0,49 & 0,58 & 1 & \\
Cooperação & 0,54 & 0,49 & 0,53 & 1 \\
Responsabilidade & 0,50 & & & \\
\hline
\end{tabular}

Nota. Fonte: Dados da pesquisa.

Os fatores encontrados corroboram resultados obtidos por Odelius e colaboradores (Odelius et al., 2011; Odelius \& Sena, 2009) e são semelhantes aos de outros estudos, como discutido a seguir.

Bergenhenegouwen et al. (1997) destacam que os valores e padrões éticos e morais abrangem a visão de mundo e/ou das outras pessoas e opiniões específicas a respeito de culturas, valores e tradições, aspectos que estão, em parte, contemplados no fator abertura à diversidade humana, uma vez que este aborda o respeito a diferenças individuais e, de alguma maneira, está associado à socialização para a profissão. Há ainda outra competência de nível intermediário considerada por esses autores, que mantém relação com esse fator: a abertura a novas ideias, que consiste em aproveitar feedbacks dos colegas como oportunidade de crescimento e aprimoramento do próprio desempenho.

Os itens abordados em abertura à diversidade humana estão associados a atitudes em relação a pessoas e a objetos (Bloom et al., 1974; Gagné \& Medsker, 1996; Pozo, 2002).

O fator habilidades sociais refere-se ao grau de assertividade das pessoas ao se posicionarem na interação com outras pessoas e nas situações com as quais se defrontam. Incluem itens relacionados à interação com outras pessoas e à formação de redes, comunicação, posicionamento de ideias, compartilhamento de experiências, reflexão, solução de problemas e resolução de conflitos. Os itens são semelhantes a metacompetências de Cheetham e Chivers (1996, 1998, 2005), às competências intermediárias de Bergenhenegouwen et al. (1997), às habilidades sociais (assertividade, autoestima, comunicação, autoexposição e autocontrole da agressividade) de Del Prette e Del Prette (2006) e às 
habilidades sociais de Pozo (2002). Porém, os itens agilidade mental, análise, auto-desenvolvimento e criatividade (Cheetham \& Chivers, 1996, 1998, 2005) não foram abordados neste estudo.

Esse fator aborda, ainda, competências semelhantes às de Haythornthwaite (2006), relativas ao trabalho em equipe e à formação de redes sociais, e as abordadas por Higuita-López et al. (2011), apresentadas na Tabela 6 .

Tabela 6

\section{Competências Necessárias a Grupos de Pesquisa Higuita-López et al. (2011)}

\begin{tabular}{ll}
\hline Associatividade & $\begin{array}{l}\text { Ter a faculdade de reconhecer o outro e respeitar suas ideias, somar esforços, } \\
\text { interagir com outras comunidades e compartilhar ideais, por meio da associação } \\
\text { de pessoas, para dar respostas coletivas a determinadas necessidades ou } \\
\text { problemas em seu contexto social. }\end{array}$ \\
Resolução de conflitos & $\begin{array}{l}\text { Resolver problemas em situações específicas, mediando as partes envolvidas } \\
\text { para obter bem-estar geral. }\end{array}$ \\
Interpessoais & $\begin{array}{l}\text { Estabelecer relações estratégicas, pessoais e cordiais com pessoas, outros } \\
\text { grupos, organizações e outros membros do ambiente. }\end{array}$ \\
Comunicativa & $\begin{array}{l}\text { Habilidade para trocar informação de forma verbal, escrita ou virtual, com } \\
\text { interlocutores com diferentes características e de acordo com as exigências de } \\
\text { cada situação. }\end{array}$
\end{tabular}

Interação com outros grupos Identificar e interagir com grupos que investigam assuntos semelhantes. de pesquisa

Nota. Fonte: elaborado pelos autores com base nos dados de Higuita-López, D., Molano-Velandia, J. H., \& RodríguezMerchán, M. F. (2011). Competencias necesarias en los grupos de investigación de la Universidad Nacional de Colombia que generan desarrollos de base tecnológica. Innovar, 21(41), 209-224.

A habilidade social, encontrada neste estudo, é semelhante também à identificada por Godoy et al. (2009) e denominada de competência social. Esses autores destacam que essa dimensão corresponde, na literatura, às competências relacionais de Zarifian (2003), às competências sociais de Fleury e Fleury (2001) e à competência pessoal ou comportamental de Cheetham e Chivers (2005), ou seja, à capacidade de adotar comportamentos apropriados e observáveis em situações relacionadas ao trabalho.

O fator cooperação refere-se à atuação colaborativa, ao compartilhamento de conhecimento e à atuação conjunta na realização das atividades. Esse fator aborda competências semelhantes às de Haythornthwaite (2006), relativas ao trabalho em colaboração, e pode ser considerado uma atitude, sob a perspectiva de Gagné e Medsker (1996) e Abbad e Borges-Andrade (2014).

O fator responsabilidade trata de atitudes pessoais em relação ao trabalho e ao compromisso com o trabalho em equipe, tal como definidos por Pozo (2002), Gagné e Medsker (1996), entre outros citados anteriormente. Refere-se ao modo como o indivíduo se posiciona na realização das atividades visando ao alcance de resultados e é semelhante a uma das competências intermediárias estabelecidas por Bergenhenegouwen et al. (1997): a aceitação de responsabilidade. Para Higuita-López et al. (2011), assumir as atividades designadas com compromisso, gerando confiança com os membros do grupo, é um dos componentes do trabalho em equipe. Nesse sentido, vale ressaltar que os quatro fatores, abertura à diversidade humana, habilidades sociais, cooperação e responsabilidade, em seu conjunto, são componentes afetivo-atitudinais de competências necessárias ao trabalho em equipe. 


\section{Conclusão}

Os objetivos propostos por esse estudo foram alcançados, uma vez que a escala desenvolvida para medir componentes atitudinais e afetivos de competências (Atitudes e Habilidades Sociais) para o Trabalho em Equipe (AHTE) apresentou parâmetros adequados e recomendados pela literatura.

Os fatores encontrados apresentam similaridade parcial com outros estudos, mas traz como contribuição o fato de pesquisar um campo relativamente pouco explorado, o de competências necessárias e desenvolvidas por estudantes de graduação e pós-graduação em grupos de pesquisa. Essas competências são identificadas na literatura como de difícil desenvolvimento, o que faz com que um instrumento que permita conhecer o domínio das mesmas possa contribuir para diagnósticos e subsidiar a definição de planos de ação de incentivo à aprendizagem colaborativa em grupos de pesquisa.

A escala desenvolvida, em conjunto com a escala de competências técnicas (Fernandez \& Odelius, 2013), poderá ser utilizada com diferentes objetivos, dependendo das instruções fornecidas no momento de coleta de dados. Entre os possíveis usos estão: processos seletivos para a identificação do domínio de competências de um candidato a participar do grupo de pesquisa, identificação de necessidade de desenvolvimento de competências ou, ainda, para a identificação de quanto o grupo contribuiu para o desenvolvimento das competências abordadas.

A escala desenvolvida e os resultados obtidos apresentam algumas limitações: (a) itens com cargas fatoriais baixas; (b) o fator responsabilidade ficou constituído por apenas dois itens, de modo que, provavelmente, não representa o construto em toda a sua complexidade ou domínio de definição.

Há ainda alguns itens identificados em estudos anteriores referentes a competências necessárias a grupos de pesquisa, como agir de maneira proativa para resolver problemas, que foram eliminados.

Para a superação das limitações identificadas, é recomendada a avaliação de necessidade de revisão da redação dos itens que apresentaram cargas fatoriais baixas e a inclusão de novos itens no fator responsabilidade.

A realização de novos estudos com análise fatorial confirmatória é recomendada, bem como uma análise comparativa com o conceito comprometimento, correlato ao constructo responsabilidade, tal como referido pelos itens do questionário descrito neste estudo.

Há também a recomendação de aplicação, em outras amostras, das escalas afetivo-atitudinais, descritas neste artigo, em conjunto com as mensuradas pelo instrumento de Fernandez e Odelius (2013), que aborda aspectos cognitivos. Esse estudo permitirá analisar prováveis correlações entre as escalas e confirmar a distinção entre os componentes cognitivos e afetivo-atitudinais de competências para o trabalho em equipe.

Novos estudos com o uso da escala validada poderão possibilitar a identificação de semelhanças e diferenças em grupos de pesquisa de uma mesma área de conhecimento, observando o grau de contribuição da participação em grupo de pesquisa para o desenvolvimento de todas as habilidades e atitudes medidas pelas escalas; a identificação de semelhanças e diferenças na aprendizagem dessas habilidades sociais e éticas entre as diferentes áreas de conhecimento; a análise do relacionamento entre o domínio dessas habilidades e variáveis demográficas tais como idade, tempo de experiência, tipo de atuação no grupo e grau de formação do participante; bem como a análise fatorial confirmatória para avaliar a hipótese de que o grau de contribuição da participação do estudante no grupo de pesquisa está correlacionado com o domínio de competências técnicas e habilidades sociais essenciais à pesquisa e à atuação profissional nos diversos campos e disciplinas científicas.

Ainda em relação a estudos futuros, é importante a análise das interações entre indivíduos com o intuito de identificar como ocorre o desenvolvimento de competências, bem como comparar o desempenho de egressos dos cursos de graduação e pós-graduação que participaram de grupos de 
pesquisa e que não participaram desse tipo de atividade durante o período de formação. Além disto, para se estudar tais efeitos e processos, poderia ser realizada a análise de redes sociais, uma vez que o estudo das mesmas facilitaria a compreensão da influência dos atributos e comportamentos dos egressos dentro do seu ambiente social e de carreira.

A aplicação das escalas descritas possibilita identificar as competências necessárias para atuação em equipe e relacioná-las com outros construtos, buscando evidências de validade de critério e de validade preditiva dessas escalas em relação a variáveis objetivas, como desempenho e resultados da equipe, mensurados em termos de indicadores de produtividade, inovação e formação de profissionais de pesquisa.

\section{Agradecimentos}

Os autores agradecem o Conselho Nacional de Desenvolvimento Científico e Tecnológico (CNPq), pelo financiamento a pesquisa. Processo: 401141/2010-1.

\section{Referências}

Abbad, G. S., \& Borges-Andrade, J. E. (2014). Aprendizagem humana em organizações de trabalho. In J. C. Zanelli, J. E. Borges-Andrade, \& A. V. B. Bastos (Orgs.), Psicologia, organizações e trabalho no Brasil (2a ed., Cap. 6, pp. 244-284). Porto Alegre: Artmed.

Abma, T. A., Nierse, C. J., \& Widdershoven, G. A. M. (2009). Patients as partners in responsive research: methodological notions for collaborations in mixed research teams. Qualitative Health Research, 19(3), 401-415. doi: 10.1177/1049732309331869

Alon, U. (2010). How to build a motivated research group. Molecular Cell, 37(2), 151-152. doi: 10.1016/j.molcel.2010.01.011

Alvarez, D., \& Vidal, M. C. R. (2001, outubro). A organização do trabalho na produção acadêmica: redes de pesquisa e estratégias de ação. Anais do Encontro Nacional de Engenharia de Produção International Congress on Industrial Engineering, Salvador, BA, Brasil, 21.

Antonello, C. S. (2006). Aprendizagem na ação revisada e sua relação com a noção de competência. Comportamento Organizacional e Gestão, 12(2), 199-220.

Bahry, C. P., \& Tolfo, S. R. (2007). Mobilização de competências nas atividades profissionais dos egressos de um programa de formação e aperfeiçoamento. Revista de Administração Pública, 41(1), 125-144. doi: 10.1590/S0034-76122007000100008

Barbosa, S. F. F., Sasso, G. T. M., \& Berns, I. (2009). Enfermagem e tecnologia: análise dos grupos de pesquisa cadastrados na plataforma Lattes do CNPq. Texto e Contexto Enfermagem, 18(3), 443448. doi: 10.1590/S0104-07072009000300006

Barrachina, L. A., Sanz, M., \& Serrat, E. (2009). Una propuesta de renovación metodológica en el marco del Espacio Europeo de Enseñanza Superior: los pequeños grupos de investigación cooperativos. REIFOP, 12(3), 111-126.

Bentler, P. M., \& Yuan, K.-H. (1996). Test of linear trend in eigenvalues of a covariance matrix with application to data analysis. British Journal of Mathematical and Statistical Psychology, 49(2), 299-312. doi: 10.1111/j.2044-8317.1996.tb01090.x 
Bentler, P. M., \& Yuan, K.-H. (1998). Test of linear trend in the smallest eigenvalues of the correlation matrix. Psychometrika, 63(2), 131-144. doi: 10.1007/BF02294771

Bergenhenegouwen, G. J., Horn, H. F. K., \& Mooijman, E. A. M. (1997). Competence development - a challenge for human resource professionals: core competences of organizations as guidelines for the development of employees. Industrial and Commercial Training, 29(2), 55-62. doi: $10.1108 / 00197859710165038$

Bloom, B. S., Krathwohl, D. R., \& Masia, B. B. (1974). Taxonomia de objetivos educacionais compêndio segundo: domínio afetivo. Porto Alegre: Globo.

Bowditch, J. L., \& Buono, A. F. (1992). Elementos de comportamento organizacional. São Paulo: Pioneira.

Brandão, H. P. (2007). Competências no trabalho: uma análise da produção científica brasileira. Estudos de Psicologia, 12(2), 149-158. doi: 10.1590/S1413-294X2007000200007

Brandão, H. P. (2008). Aprendizagem e competências nas organizações: uma revisão crítica de pesquisas empíricas. GESTÃO.Org - Revista Eletrônica de Gestão Organizacional, 6(3), 321-342.

Brandão, H. P., \& Borges-Andrade, J. E. (2007). Causas e efeitos da expressão de competências no trabalho: para entender melhor a noção de competência. Revista de Administração Mackenzie, $8(3), 32-49$.

Brandão, H. P., \& Guimarães, T. A. (2001). Gestão de competências e gestão de desempenho: tecnologias distintas ou instrumentos de um mesmo construto? Revista de Administração de Empresas, 41(1), 8-15. doi: 10.1590/S0034-75902001000100002

Cassep-Borges, V., Balbinotti, M. A. A., \& Teodoro, M. L. M. (2010). Tradução e validação de conteúdo: uma proposta para a adaptação de instrumentos. In L. Pasquali (Ed.), Instrumentação psicológica: fundamentos e práticas (pp. 506-520). Porto Alegre: Artmed.

Cheetham, G., \& Chivers, G. (1996). Towards a holistic model of professional competence. Journal of European Industrial Training, 20(5), 20-30. doi: 10.1108/03090599610119692

Cheetham, G., \& Chivers, G. (1998). The reflective (and competente) practitioner. A model of professional competence with seeks to harmonise the reflective practitioner and competence based approaches. Journal of European Industrial Training, 22(7), 267-276.

Cheetham, G., \& Chivers, G. (2005) Professions, competence and informal learning. Cheltenham, UK: Edward Elgar.

Creswell, J. W. (2010). Projeto de pesquisa: métodos qualitativo, quantitativo e misto (3a ed.). Porto Alegre: Artmed/Bookman.

Del Prette, A., \& Del Prette, Z. A. P. (2006). Habilidades sociais: conceitos e campo teórico- prático. Recuperado de http://betara.ufscar.br:8080/pesquisa/rihs/armazenagem/pdf/artigos/habilidadessociais-conceitos-e-campo-teorico-pratico

$\mathrm{Du}, \mathrm{F}$. (2009). Building action research teams: a case of struggles and successes. Journal of Cases in Educational Leadership, 12(8), 8-18. doi: 10.1177/1555458909336893

Durand, T. (1997, Juin). Savoir, savoir-faire et savoir-être: repenser les compétences de l'entreprise. Actes de Conférence de l'Association Internationale de Management Stratégique, Montréal, Quebec, CA, 6.

Dutra, J. S. (2004). Competências: conceito e instrumentos para a gestão de pessoas na empresa moderna. São Paulo: Atlas. 
Erdmann, A. L., \& Lanzoni, G. M. M. (2008). Características dos grupos de pesquisa da enfermagem brasileira certificados pelo CNPq de 2005 a 2007. Revista de Enfermagem, 12(2), 316-322. doi: $10.1590 / \mathrm{S} 1414-81452008000200018$

Fernandez, F. F., \& Odelius, C. C. (2013). Validação de uma escala de domínio de competências em grupos de pesquisa. Revista de Administração, 12(2), 81-97.

Fleury, M. T. L., \& Fleury, A. C. C. (2001). Construindo o conceito de competência [Edição Especial]. Revista de Administração Contemporânea, 5, 193-196. doi: 10.1590/S1415-65552001000500010

Freitas, I. A., \& Brandão, H. P. (2006). Trilhas de aprendizagem como estratégia para desenvolvimento de competências. In J. E. Borges-Andrade, G. S. Abbad, \& L. Mourão (Orgs.), Treinamento, desenvolvimento e educação em organizações e trabalho: fundamentos para a gestão de pessoas (pp. 97-113). Porto Alegre: Artmed/Bookman.

Gagné, R. M., \& Medsker, K. L. (1996). The conditions of learning: training applications (4a ed.). Belmont: Wadsworth Group/Thompson Learning.

Garavan, T. N., \& McGuire, D. (2001). Competencies and workplace learning: some reflections on the rhetoric and the reality. Journal of Workplace Learning, 13(4), 144-164. doi: $10.1108 / 13665620110391097$

Gaviria Velásquez, M. M., Mejía Correa, A. M., \& Henao Henao, D. L. (2007). Gestión del conocimiento en los grupos de investigación de excelencia de la Universidad de Antioquia. Revista Interamericana de Bibliotecología, 30(2), 137-163.

Godoy, A. S., \& Antonello, C. S. (2009). Competências individuais adquiridas durante os anos de graduação de alunos do curso de administração de empresas. Revista de Ciências da Administração, 11(23), 11-29. doi: 10.5007/2175-8077.2009v11n23p157

Godoy, A. S., Antonello, C. S., Bido, D. S., \& Silva, D. (2009). O desenvolvimento das competências de alunos formandos do curso de administração: um estudo de modelagem de equações estruturais. Revista de Administração, 44(3), 265-278.

González-Alcaide, G., Aleixandre-Benavent, R., \& Granda-Orive, J. I. (2010). Caracterización bibliométrica y temática de los grupos de investigación de Archivos de Bronconeumología (20032007). Archivos de Bronconeumología, 46(2), 78-84. doi: 10.1016/j.arbres.2009.10.014

Hamui Sutton, M. (2010a). Estructura de organización en la trayectoria de dos grupos de investigación científica de ciencias básicas de la salud en la generación de conocimiento. Revista Mexicana de Investigación Educativa, 15(46), 713-738.

Hamui Sutton, M. (2010b). Ethos en la trayectoria de dos grupos de investigación científica de ciencias básicas de la salud. Revista de la Educación Superior, 39(154), 51-74.

Haythornthwaite, C. (2006). Learning and knowledge networks in interdisciplinary collaborations. Journal of the American Society for Information Science and Technology, 57(8), 1079-1092. doi: 10.1002/asi.20371

Higuita-López, D., Molano-Velandia, J. H., \& Rodríguez-Merchán, M. F. (2011). Competencias necesarias en los grupos de investigación de la Universidad Nacional de Colombia que generan desarrollos de base tecnológica. Innovar, 21(41), 209-224.

Jones, L., \& Moore, R. (2008). La apropiación del significado de competencia: el movimiento de la competencia, la nueva derecha y el proyecto de cambio cultural. Revista de Currículum y Formación del Profesorado, 12(3), 1-20. 
Jubb, R., \& Robotham, D. (1997). Competence in management development: challenging the myths. Journal of European Industrial Training, 21(5), 171-175. doi: 10.1108/03090599710171422

King, A. W., Fowler, S. W., \& Zeithaml, C. P. (2002). Competências organizacionais e vantagem competitiva: o desafio da gerência intermediária. Revista de Administração de Empresas, 42(1), 36-49. doi: 10.1590/S0034-75902002000100005

Le Boterf, G. (2003). Desenvolvendo a competência dos profissionais. Porto Alegre: Artmed.

Leitão, S. S. (2010). A importância do desenvolvimento de competências para a mudança organizacional. Gestão Contemporânea, 7(7), 245-268.

Martin-Baró, I. (1983). Acción e ideologia: psicologia social desde centroamérica. San Salvador: UCA.

Mauthner, N. S., \& Doucet, A. (2008). Knowledge once divided can be hard to put together again: an epistemological critique of collaborative and team-based research practices. Sociology, 42(5), 971-985. doi: 10.1177/0038038508094574

McClelland, D. (1973). Testing for competence rather than for intelligence. American Psycologist, 28(1), 1-14. doi: 10.1037/h0034092

McMullan, M., Endacott, R., Gray, M. A., Jasper, M., Miller, C. M. L., Scholes, J., \& Webb, C. (2003). Portfolios and assessment of competence: a review of the literature. Journal of Advanced Nursing, 41(3), 283-294. doi: 10.1046/j.1365-2648.2003.02528.x

Millward, L. J., Banks, A., \& Riga, K. (2010). Effective self-regulating teams: a generative psychological approach. Team Performance Management, 16(1/2), 50-73. doi: $10.1108 / 13527591011028924$

Moura, G. R., Leader, T., Pelletier, J., \& Abrams, D. (2008). Prospects for group processes and intergroup relations research: a review of 70 years' progress. Group Processes \& Intergroup Relations, 11(4), 575-596. doi: 10.1177/1368430208095406

Nierse, C. J., Schipper, K., Zadelhoff, E., van, Griendt, J., van de, \& Abma, T. A. (2011). Collaboration and co-ownership in research: dynamics and dialogues between patient research partners and professional researchers in a research team. Health Expectations, 15(3), 242-254. doi: 10.1111/j.1369-7625.2011.00661.x

Odelius, C. C., Abbad, G. S., Resende-Júnior, P. C., Sena, A. C., \& Ono, R. N. (2010, setembro). Grupos de pesquisa: atividades, competências e processos de aprendizagem. Anais do Encontro Nacional da Associação Nacional de Pós-Graduação e Pesquisa em Administração, Rio de Janeiro, RJ, Brasil, 34.

Odelius, C. C., Abbad, G. S, Resende-Júnior, P. C., Sena, A. C., Viana, C. R., Freitas, T. L., \& Santos, T. C. N. (2011). Processos de aprendizagem, competências aprendidas, funcionamento, compartilhamento e armazenagem de conhecimentos em grupos de pesquisa. Caderno EBAPE, 9(1), 199-220. doi: 10.1590/S1679-39512011000100012

Odelius, C. C., \& Sena, A. C. (2009). Atuação em grupos de pesquisa: competências e processos de aprendizagem. Revista de Administração, 8(4), 13-31.

Olsson, U. (1979). Maximum likelihood estimation of the polychoric correlation coefficient. Psychometrika, 44(4), 443-460. doi: 10.1007/BF02296207

Parry, S. B. (1996). The quest for competencies. Training, 33(7), 48-54.

Pasquali, L. (2008). Análise fatorial para pesquisadores. Brasília: LabPAM, UnB. 
Pasquali, L. (2010). Instrumentação psicológica: fundamentos e práticas. Porto Alegre: Artmed.

Pepe, V. L. E., Noronha, A. B. M., Figueiredo, T. A., Souza, A. A. L. Oliveira, C. V. S., \& Pontes-Júnior, D. M. (2010). A produção científica e grupos de pesquisa sobre vigilância sanitária no CNPq. Ciência e Saúde Coletiva, 15(3), 3341-3350. doi: 10.1590/S1413-81232010000900009

Peres-dos-Santos, L. F. B., \& Laros, J. A. (2007). Avaliação da prática pedagógica do professor do ensino superior. Estudos em Avaliação Educacional, 18(36), 75-96. Recuperado de http://www.fcc.org.br/pesquisa/publicacoes/eae/arquivos/1359/1359.pdf.

Pozo, J. I. (1999). Aprendices y maestros. La nueva cultura del aprendizaje. Madrid: Alianza.

Pozo, J. I. (2002). Aprendizes e mestres: a nova cultura da aprendizagem. Porto Alegre: Artmed.

Restrepo, M. I., \& Villegas, J. G. (2007). Clasificación de grupos de investigación colombianos aplicando análisis envolvente de datos. Revista Facultad de Ingeniería Universidad de Antioquia, (42), 105-119.

Richardson, R. J. (2010). Pesquisa social - métodos e técnicas (3a ed.). São Paulo: Atlas.

Rigdon, E. E., \& Ferguson, C. E., Jr. (1991). The performance of the polychoric correlation coefficient and selected fitting functions in confirmatory factor analysis with ordinal data. Journal of Marketing Research, 28(4), 491-497. doi: 10.2307/3172790

Rindskopf, D. (1984). Structural equation models empirical identification, Heywood cases, and related problems. Sociological Methods \& Research, 13(1), 109-119. doi: $10.1177 / 0049124184013001004$

Riquelme, G. C., \& Langer, A. (2010). Capacidades de los grupos de docencia e investigación en la circulación y producción del conocimiento: el caso de tres universidades argentinas. Revista de la Educación Superior, 39(154), 19-50.

Rizo, V., \& Eduardo, F. (2010). Modelo de gestión del conocimiento para medir la capacidad productiva en grupos de investigación. Ciencia, Docencia y Tecnología, (41), 101-125.

Rocha, D., \& Deusdará, B. A. (2005). Análise de conteúdo e análise do discurso: aproximações e afastamentos na (re)construção de uma trajetória. ALEA Estudos Neolatinos, 7(2), 305-322. doi: 10.1590/S1517-106X2005000200010

Rowe, C. (1995). Clarifying the use of competence and competency models in recruitment, assessment and staff development. Industrial and Commercial Training, 27(11), 12-17. doi: $10.1108 / 00197859510100257$

Skinner, B. F. (1987). Upon further reflection. Englewood Cliffs, NJ: Prentice Hall.

Stevens, S. S. (1946). On the theory of scales of measurement. Science, 103(2684), 677-680. doi: $10.1126 /$ science. 103.2684 .677

Todorov, J. C. (1989). Psicologia como estudo de interações. Psicologia: Teoria e Pesquisa, 7(3), 347356.

Weijden, I., van der, Gilder, D. de, Groenewegen, P., \& Klasen, E. (2008). Implications of managerial control on performance of Dutch academic (bio)medical and health research groups. Research Policy, 37(9), 1616-1629. doi: 10.1016/j.respol.2008.06.007

Williams, E. A., Scandura, T. A., \& Gavin, M. (2009). Understanding team-level career mentoring by leaders and its effects on individual team-source learning: the effects of intra-group processes. Human Relations, 62(11), 1635-1666. doi: 10.1177/0018726709346375 
Zarifian, P. (2001). Objetivo competência: por uma nova lógica. São Paulo: Atlas.

Zarifian, P. (2003). O modelo da competência: trajetória histórica, desafios atuais e propostas. São Paulo: Senac.

Zoski, K., \& Jurs, S. (1993). Using multiple regression to determine the number of factors to retain in factor analysis. Multiple Linear Regression Viewpoints, 20(1), 5-9.

Zoski, K., \& Jurs, S. (1996). An objective counterpart to the visual scree test for factor analysis: the standard error scree test. Educational and Psychological Measurement, 56(3), 443-451. doi: $10.1177 / 0013164496056003006$

Zumbo, B. D., Gadermann, A. M., \& Zeisser, C. (2007). Ordinal versions of coefficients alpha and theta for likert rating scales. Journal of Modern Applied Statistical Methods, 6(1), 21-29.

\title{
Dados dos Autores
}

\author{
Catarina Cecília Odelius \\ Campus Universitário Darcy Ribeiro, Prédio da FACE, Asa Norte, 70910-900, Brasília, DF, Brasil: E-mail: codelius@unb.br, \\ codelius@gmail.com \\ Rafael Nishino Ono \\ Campus Universitário Darcy Ribeiro, Prédio da FACE, Asa Norte, 70910-900, Brasília, DF, Brasil: E-mail: \\ rafael.admunb@gmail.com \\ Gardênia da Silva Abbad \\ Campus Universitário Darcy Ribeiro, Prédio da FACE, Asa Norte, 70910-900, Brasília, DF, Brasil: E-mail: \\ gardenia.abbad@gmail.com \\ Pedro Henrique Melo Albuquerque \\ Campus Universitário Darcy Ribeiro, Prédio da FACE, Asa Norte, 70910-900, Brasília, DF, Brasil: E-mail: pedroa@unb.br
}

